\title{
MACROSCOPIC TURBULENCE MODEL ADJUSTMENT FOR A POROUS MEDIUM MODELED AS AN INFINITE ARRAY OF TRANSVERSALLY-DISPLACED ELLIPTIC RODS
}

\author{
M. H. J. Pedras*, \\ M. J. S. de Lemos ${ }^{\S}$, \\ *Instituto de Pesquisa e Desenvolvimento \\ IP\&D, UNIVAP, Av. Shishima Hifumi, 2911- \\ São José dos Campos-SP, Brazil \\ pedras@univap.br \\ ABSTRACT \\ Through the volumetric averaging of the microscopic transport equations for the turbulent \\ kinetic energy, $k$, and its dissipation rate, $\varepsilon$, a macroscopic model was proposed for flow in \\ porous media (Pedras and de Lemos, IJHMT 44 (6) 2001). As an outcome of the volume \\ averaging process, additional terms appeared in the equations for $k$ and $\varepsilon$. These terms \\ were here adjusted assuming the porous structure to be modeled as an infinity array of \\ transversal elliptic rods. Such an adjustment was obtained by numerically solving the \\ microscopic flow governing equations, using a low and high Reynolds formulation, in the \\ periodic cell composing the infinite medium. Different porosity and aspect ratios were \\ investigated. The adjusted model was compared against others types of rods found in the \\ literature, showing similar results. \\ dos Campos-SP, Brazil \\ delemos@mec.ita.br \\ Keywords: porous media, turbulent flows, periodic boundary conditions, low Reynolds \\ $k$ - $\varepsilon$ model, macroscopic turbulence model.
}

\section{INTRODUCTION}

Due to its great application in the industry and science, the study of the flow in porous media has received great attention lately. Engineering systems based on fluidized bed combustion, enhanced oil reservoir recovery, combustion in an inert porous matrix, underground spreading of chemical waste and chemical catalytic reactors are just a few examples of such applications.

Mathematical treatment of flow in porous media (Darcy 1856, Forchheimer 1901, Brinkman 1947,Ward 1964, Whitaker 1969, Bear 1972, Vafai and Tien 1981, Hsu and Cheng 1990, Pedras and de Lemos 1999, Pedras and de Lemos 2000, de Lemos and Pedras 2000, Pedras and de Lemos 2001a) uses the notion of a Representative Elementary Volume (REV, Fig. 1) for which governing equations are derived. These models, based on the macroscopic point of view, lose details on the flow pattern inside the REV and, together with ad-hoc information, provide global flow properties such as average velocities and temperatures.

Such flows can also be analyzed by modeling the topology of the porous medium and resolving the microscopic flow equation in the liquid phase. This treatment reveals the microscopic flow structure and was used by Kuwahara et al. (1998) who adopted a spatially periodic array of square rods and by Pedras and de Lemos $(2001 \mathrm{~b}, \mathrm{c}, \mathrm{d})$ who used cylindrical and longitudinal elliptic rods instead. A discussion about the relationship between these two treatments (macroscopic and microscopic) is found in Pedras and de Lemos (1998) and de Lemos and Pedras (2001).

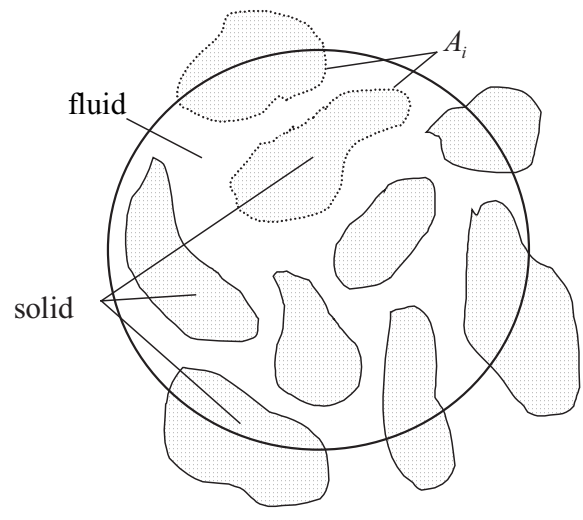

Fig. 1 - Representative elementary volume (REV).

Because of these two different points of view, the existence or not of turbulence in flow in porous media is still in debate (Antohe and Lage 1997, Kuwahara et al. 1998, Nakayama and Kuwahara 1999, Pedras and de Lemos 1999, Pedras and de Lemos 2000, de Lemos and Pedras 2000, de Lemos and Pedras 2001, Pedras and de Lemos 2001a). Considering available experimental work reporting the existence of turbulence in such media (Macdonald et al. 1979, Kececiooglu and Jiang 1994, Takatsu and Masuoka 1998), de Lemos and Pedras (2000) and Pedras and de Lemos (2001) proposed a macroscopic turbulence model through the volumetric averaging of the microscopic $k-\varepsilon$ equations. This macroscopic model, in contrast with the microscopic $k-\varepsilon$ equations, presents two extra terms that represent extra transport/production of turbulence energy due to the presence of the solid material inside the integrated volume.

The aim of the present contribution is to adjust these new terms by numerically solving the flow field within a spatially periodic array of transversal elliptic rods. Both Low and High Re $k-\varepsilon$ models are employed. 
Additional constants are determined and, with the macroscopic turbulence model so adjusted, the turbulence kinetic energy and its dissipation rate are compared with existing macroscopic flow solutions presented in the literature.

\section{MACROSCOPIC $\boldsymbol{k}-\varepsilon$ EQUATIONS}

de Lemos and Pedras (2000) and Pedras and de Lemos (2001a) have applied the volume-averaging operator to the microscopic $k-\varepsilon$ equations and proposed the following macroscopic $k-\varepsilon$ equations:

$$
\begin{array}{r}
\rho\left[\frac{\partial}{\partial t}\left(\phi\langle k\rangle^{i}\right)+\nabla \cdot\left(\overline{\mathbf{u}}_{D}\langle k\rangle^{i}\right)\right]=\nabla \cdot\left[\left(\mu+\frac{\mu_{t_{\phi}}}{\sigma_{k}}\right) \nabla\left(\phi\langle k\rangle^{i}\right)\right] \\
-\rho\left\langle\overline{\mathbf{u}^{\prime} \mathbf{u}^{\prime}}\right\rangle^{i}: \nabla \overline{\mathbf{u}}_{D}+c_{k} \rho \phi \frac{\langle k\rangle^{i}\left|\overline{\mathbf{u}}_{D}\right|}{\sqrt{K}}-\rho \phi\langle\varepsilon\rangle^{i} \\
\rho\left[\frac{\partial}{\partial t}\left(\phi\langle\varepsilon\rangle^{i}\right)+\nabla \cdot\left(\overline{\mathbf{u}}_{D}\langle\varepsilon\rangle^{i}\right)\right]=\nabla \cdot\left[\left(\mu+\frac{\mu_{t_{\phi}}}{\sigma_{\varepsilon}}\right) \nabla\left(\phi\langle\varepsilon\rangle^{i}\right)\right] \\
+c_{1 \varepsilon}\left(-\rho\left\langle\overline{\mathbf{u}^{\prime} \mathbf{u}^{\prime}}\right\rangle^{i}: \nabla \overline{\mathbf{u}}_{D}\right) \frac{\langle\varepsilon\rangle^{i}}{\langle k\rangle^{i}} \\
+c_{2 \varepsilon} \rho \phi\left\{c_{k} \frac{\langle\varepsilon\rangle^{i}\left|\overline{\mathbf{u}}_{D}\right|}{\sqrt{K}}-\frac{\langle\varepsilon\rangle^{i^{2}}}{\langle k\rangle^{i}}\right\}
\end{array}
$$

with,

$$
\begin{aligned}
& -\rho \phi\left\langle\overline{\left.{\mathbf{\mathbf { u } ^ { \prime }}}^{\prime}\right\rangle^{i}}=\mu_{t_{\phi}} 2\langle\overline{\mathbf{D}}\rangle^{\mathbf{v}}-\frac{2}{3} \phi \rho\langle k\rangle^{i} \mathbf{I}\right. \\
& \mu_{t_{\phi}}=\rho c_{\mu} \frac{\langle k\rangle^{i^{2}}}{\langle\varepsilon\rangle^{i}}
\end{aligned}
$$

where $c_{k}, \quad c_{1 \varepsilon}, \quad c_{2 \varepsilon}$ and $c_{\mu}$ are non-dimensional constants.

For macroscopic fully developed unidimensional flow in isotropic and homogeneous media, the limiting values for $\langle k\rangle^{i}$ and $\langle\varepsilon\rangle^{i}$ are given by $k_{\phi}$ and $\varepsilon_{\phi}$, respectively. In this limiting condition, Eqs. (1) and

(2) reduce to:

$$
\left.\begin{array}{l}
\langle\varepsilon\rangle^{i}=\varepsilon_{\phi}=c_{k} \frac{k_{\phi}\left|\overline{\mathbf{u}}_{D}\right|}{\sqrt{K}} \\
\frac{\langle\varepsilon\rangle^{i^{2}}}{\langle k\rangle^{i}}=c_{k} \frac{\varepsilon_{\phi}\left|\overline{\mathbf{u}}_{D}\right|}{\sqrt{K}}
\end{array}\right\} \Rightarrow\langle k\rangle^{i}=k_{\phi}
$$

or in the following dimensionless form,

$$
\frac{\varepsilon_{\phi} \sqrt{K}}{\left|\overline{\mathbf{u}}_{D}\right|^{3}}=c_{k} \frac{k_{\phi}}{\left|\overline{\mathbf{u}}_{D}\right|^{2}}
$$

The coefficient $c_{k}$ was adjusted in this limiting condition and for the spatially periodic cell shown in Fig. 2. This geometry represents a model for the fine porous structure. The flow was assumed to enter through the left aperture so that symmetric and periodic boundary conditions were applied. Values of $k_{\phi}$ and $\varepsilon_{\phi}$ were obtained by integrating the microscopic flow field for Reynolds number, $\operatorname{Re}_{H}=\left|\overline{\mathbf{u}}_{D}\right| H / v$, from $10^{4}$ to $10^{6}$.
The porosity, given by $\phi=1-a b \pi / H^{2}$, was varied from 0.70 to 0.90 .

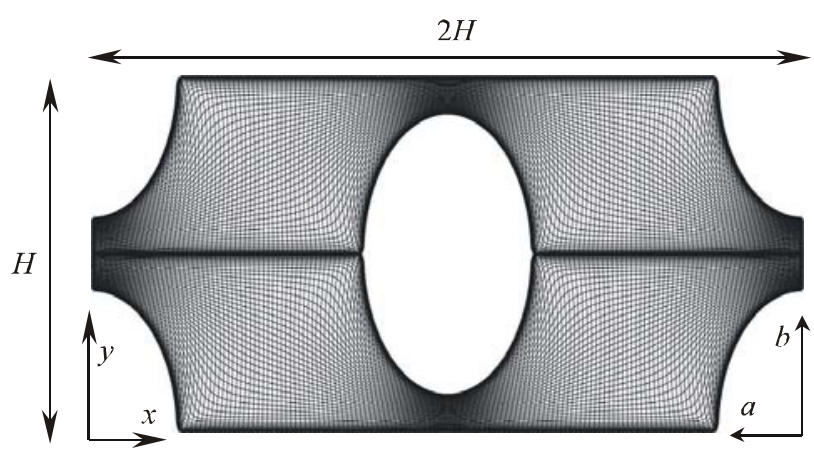

Fig. 2 - Periodic cell and elliptically generated grid $(a / b=3 / 5)$.

\section{MICROSCOPIC FLOW EQUATIONS}

In the numeric model, the following microscopic transport equations were used, where barred quantities represent time-averaged components and primes indicate turbulent fluctuations:

Continuity equation

$$
\nabla \cdot \overline{\mathbf{u}}=0
$$

Momentum equation

$$
\nabla \cdot(\rho \overline{\mathbf{u}} \overline{\mathbf{u}})=-\nabla \bar{p}+\nabla \cdot\left\{\mu\left[\nabla \overline{\mathbf{u}}+(\nabla \overline{\mathbf{u}})^{T}\right]-\rho \overline{\mathbf{u}^{\prime} \mathbf{u}^{\prime}}\right\}
$$

$k$ equation

$$
\nabla \cdot(\rho \overline{\mathbf{u}} k)=\nabla \cdot\left[\left(\mu+\frac{\mu_{t}}{\sigma_{k}}\right) \nabla k\right]-\rho \overline{\mathbf{u}^{\prime} \mathbf{u}^{\prime}}: \nabla \overline{\mathbf{u}}-\rho \varepsilon
$$

$\varepsilon$ equation

$$
\begin{aligned}
\nabla \cdot(\rho \overline{\mathbf{u}} \varepsilon)= & \nabla \cdot\left[\left(\mu+\frac{\mu_{t}}{\sigma_{\varepsilon}}\right) \nabla \varepsilon\right] \\
& +\left[C_{1}\left(-\rho \overline{\mathbf{u}^{\prime} \mathbf{u}^{\prime}}: \nabla \overline{\mathbf{u}}\right)-C_{2} f_{2} \rho \varepsilon\right] \frac{\varepsilon}{k}
\end{aligned}
$$

Also, the Boussinesq's concept for Reynolds stresses is given by,

$$
-\rho \overline{\mathbf{u}^{\prime} \mathbf{u}^{\prime}}=\mu_{t}\left[\nabla \overline{\mathbf{u}}+(\nabla \overline{\mathbf{u}})^{T}\right]-\frac{2}{3} \rho k \mathbf{I}
$$

where the turbulent viscosity is

$$
\mu_{t}=\rho C_{\mu} f_{\mu} \frac{k^{2}}{\varepsilon}
$$

In the above equation set $\sigma_{k}, \sigma_{\varepsilon}, C_{1}, C_{2}$ and $C_{\mu}$ are dimensionless constants whereas $f_{2}$ and $f_{\mu}$ are damping functions.

The use, in this work, of the low and high Re $k$ $\varepsilon$ models is justified by the fact that the turbulent flow in porous media occurs for Reynolds numbers (based on the pore) relatively low. To account for the low Reynolds effects, the following damping functions were adopted (Abe et al. 1992): 


$$
\begin{aligned}
& f_{2}=\left\{1-\exp \left[-\frac{(v \varepsilon)^{0.25} n}{3.1 v}\right]\right\}^{2} \times\left\{1-0.3 \exp \left[-\left(\frac{\left(k^{2} / v \varepsilon\right)}{6.5}\right)^{2}\right]\right\} \\
& f_{\mu}=\left\{1-\exp \left[-\frac{(v \varepsilon)^{0.25} n}{14 v}\right]\right\}^{2} \\
& \times\left\{1+\frac{5}{\left(k^{2} / v \varepsilon\right)^{0.75}} \exp \left[-\left(\frac{\left(k^{2} / v \varepsilon\right)}{200}\right)^{2}\right]\right\}
\end{aligned}
$$

where $n$ is the coordinate normal to the wall. The model constants are given as follows,

$$
C_{\mu}=0.09, C_{1}=1.5, C_{2}=1.9, \sigma_{k}=1.4, \sigma_{\varepsilon}=1.3 .
$$

For the high Re model the standard constants of Launder and Spalding (1974) were used.

With the assumption of macroscopic fully developed uni-dimensional flow, the following boundary conditions for the periodic cell (Fig. 2) were adopted:

at the walls, $\overline{\mathbf{u}}=0, k=0$ and $\varepsilon=v \frac{\partial^{2} k}{\partial n^{2}}$,

on $x=0$ and $x=2 H$ (periodic boundaries),

$$
\begin{aligned}
& \left.\bar{u}\right|_{x=0}=\left.\bar{u}\right|_{x=2 H},\left.\bar{v}\right|_{x=0}=\left.\bar{v}\right|_{x=2 H}, \\
& \left.k\right|_{x=0}=\left.k\right|_{x=2 H},\left.\varepsilon\right|_{x=0}=\left.\varepsilon\right|_{x=2 H},
\end{aligned}
$$

on $y=0$ and $y=H / 2$ symmetrical lines,

$$
\frac{\partial \bar{u}}{\partial y}=\frac{\partial \bar{v}}{\partial y}=\frac{\partial k}{\partial y}=\frac{\partial \varepsilon}{\partial y}=0 .
$$

where $\bar{u}$ and $\bar{v}$ are components of $\overline{\mathbf{u}}$.

\section{NUMERICAL MODEL}

The governing equations were discretized using the finite volume procedure (Patankar, 1980). The SIMPLE algorithm for the pressure-velocity coupling was adopted to correct both the pressure and the velocity fields. Process starts with the solution of the two momentum equations. Then the velocity field is adjusted in order to satisfy the continuity principle. This adjustment is obtained by solving the pressure correction equation. After that, the turbulence model equations are relaxed to update the $k$ and $\varepsilon$ fields. This iteration sequence is repeated until convergence is achieved. Details on the numerical discretization can be found in Rocamora and de Lemos (1998) and in Pedras and de Lemos (2001b).

For the low Re model, the node adjacent to the wall requires that $u_{\tau} n / v \leq 1$. To accomplish this requirement, the grid needs a great number of points close to the wall leading to computational meshes of large sizes. In order to minimize this problem, all calculations were made in half of the periodic cell $(2 \mathrm{H} \times \mathrm{H} / 2)$ and according to the boundary condition Eq. (19). The use of the symmetry boundary condition reduces the grid to $300 \times 100$ nodes. A highly nonuniform grid arrangement was employed with concentration of nodes close to the wall.

\section{RESULTS AND DISCUSSION}

A total of eighteen runs were carried out being six for laminar flow, six with the low Re model and six using the high $\mathrm{Re}$ theory. Table 1 summarizes the integrated results (volumetric averaging over the periodic cell) obtained for turbulent flow. The medium permeability was calculated using the procedure adopted by Kuwahara et al. (1998).

Figure 3 presents velocity, pressure, $k$ and $\varepsilon$ fields for $\operatorname{Re}_{H}=1.67 \times 10^{5}$ (low $\operatorname{Re}$ model) and $\phi=0.70$. It is observed that the flow accelerates in the upper and lower passages around the ellipse and separates at the back. As porosity decreases maintaining $\mathrm{Re}_{H}$ constant, or say, reducing the flow passage and

\begin{tabular}{|c|c|c|c|c|c|c|}
\hline & $\begin{array}{c}\text { Medium } \\
\text { permeability }\end{array}$ & $\operatorname{Re}_{H}$ & $k-\varepsilon$ model & $\left|\overline{\mathbf{u}}_{D}\right|$ & $\langle k\rangle^{i}$ & $\langle\varepsilon\rangle^{i}$ \\
\hline \multirow{4}{*}{$\phi=0.70$} & \multirow{4}{*}{$K=2.31 \mathrm{E}-05$} & $1.67 \mathrm{E}+04$ & Low & $2.51 \mathrm{E}-01$ & $1.22 \mathrm{E}-01$ & $1.67 \mathrm{E}+00$ \\
\hline & & $1.67 \mathrm{E}+05$ & low & $2.51 \mathrm{E}+00$ & $1.10 \mathrm{E}+01$ & $1.53 \mathrm{E}+03$ \\
\hline & & $1.67 \mathrm{E}+05$ & high & $2.51 \mathrm{E}+00$ & $1.12 \mathrm{E}+01$ & $1.58 \mathrm{E}+03$ \\
\hline & & $1.67 \mathrm{E}+06$ & high & $2.51 \mathrm{E}+01$ & $1.16 \mathrm{E}+03$ & $1.58 \mathrm{E}+06$ \\
\hline \multirow{4}{*}{$\phi=0.80$} & \multirow{4}{*}{$K=8.69 \mathrm{E}-05$} & $1.67 \mathrm{E}+04$ & low & $2.51 \mathrm{E}-01$ & 6.10E-02 & 4.68E-01 \\
\hline & & $1.67 \mathrm{E}+05$ & low & $2.51 \mathrm{E}+00$ & $4.60 \mathrm{E}+00$ & $3.73 \mathrm{E}+02$ \\
\hline & & $1.67 \mathrm{E}+05$ & high & $2.51 \mathrm{E}+00$ & $5.40 \mathrm{E}+00$ & $4.23 \mathrm{E}+02$ \\
\hline & & $1.67 \mathrm{E}+06$ & high & $2.51 \mathrm{E}+01$ & $5.61 \mathrm{E}+02$ & $4.27 \mathrm{E}+05$ \\
\hline \multirow{4}{*}{$\phi=0.90$} & \multirow{4}{*}{$K=2.32 \mathrm{E}-04$} & $1.67 \mathrm{E}+04$ & low & $2.51 \mathrm{E}-01$ & $3.10 \mathrm{E}-02$ & $1.80 \mathrm{E}-01$ \\
\hline & & $1.67 \mathrm{E}+05$ & low & $2.51 \mathrm{E}+00$ & $2.36 \mathrm{E}+00$ & $1.57 \mathrm{E}+02$ \\
\hline & & $1.67 \mathrm{E}+05$ & high & $2.51 \mathrm{E}+00$ & $2.24 \mathrm{E}+00$ & $1.29 \mathrm{E}+02$ \\
\hline & & $1.67 \mathrm{E}+06$ & high & $2.51 \mathrm{E}+01$ & $2.75 \mathrm{E}+02$ & $1.78 \mathrm{E}+05$ \\
\hline
\end{tabular}
increasing the local fluid speed, the integrated turbulence kinetic energy , $\langle k\rangle^{i}$, increases (see Tab.1). In others words, for a fixed mass flow rate through the bed, a decrease in porosity implies in accentuated velocity gradients which, in turn, result in larger production rates of $k$ due to steep velocity gradients within the fluid.

Table 1 - Summary of the integrated results. 

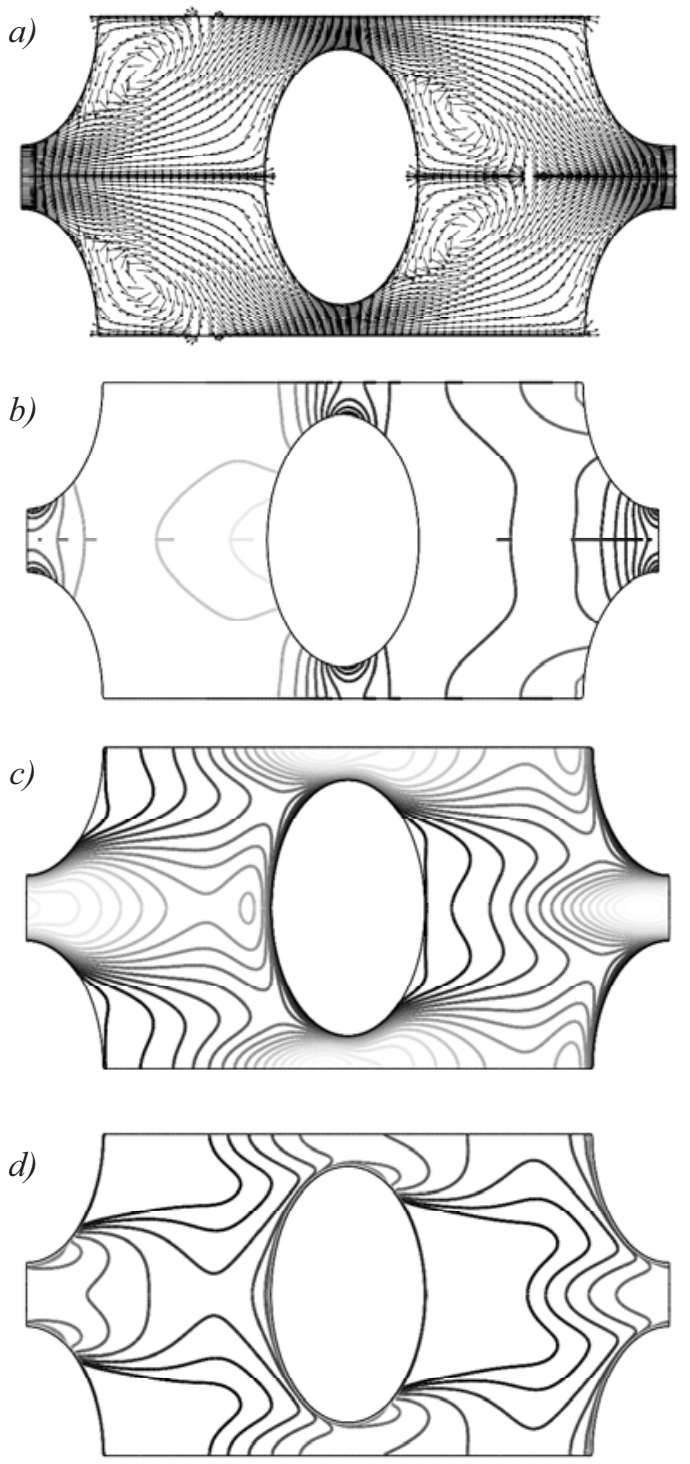

Fig. 3 - Microscopic results at $\operatorname{Re}_{H}=1.67 \times 10^{5}$ and $\phi=0.70: a)$ velocity, b) pressure, c) $k$ and $d$ ) $\varepsilon$.

Furthermore, the increment of the fluid momentum close to the walls as porosity is reduced, for the same $\left|\overline{\mathbf{u}}_{D}\right|$, reduces also the size of the recirculating bubble behind the transversal elliptic rod. This effect was also verified by Pedras and de Lemos $(2001 b, c)$ for an array of cylindrical and longitudinal elliptic rods, respectively, and by Kuwahara et al. (1998) for an array of square cylinders.

In the remainder fields, it is verified that the pressure increases at the front of the ellipse and decreases at the upper and lower faces. The turbulence kinetic energy is high at the front, on the top and below the bottom of the ellipse. The dissipation rate of $k$ presents a behavior similar to the turbulence kinetic energy.

It is also interesting to point out that for the same $\phi$ and $\operatorname{Re}_{H}$ the integrated values shown in Tab. 1 for $\langle k\rangle^{i}$ are at same level of those obtained for square rods (Nakayama and Kuwahara 1999) and greater than those obtained for cylindrical and longitudinal elliptic rods (Pedras and de Lemos 2001b,c,d). Apparently, sharper passages in between transversal elliptic rods contributes for enhancing sudden flow acceleration within the flow, enhancing then overall velocity gradients and, consequently, increasing production rates $\langle k\rangle^{i}$.

\section{CONSTANT $\mathrm{C}_{K}$ FOR THE MACROSCOPIC MODEL}

Once the intrinsic values of $k_{\phi}$ and $\varepsilon_{\phi}$ were obtained, they were plugged into Eq. (6). The value of $c_{k}$ equal to 0.28 was found by noting the collapse of all data into the straight line shown in Fig. 4. The figure also shows data of Pedras and de Lemos (2001a,c) and Nakayama and Kuwahara (1999) for cylindrical, longitudinal elliptic and square rods, respectively.

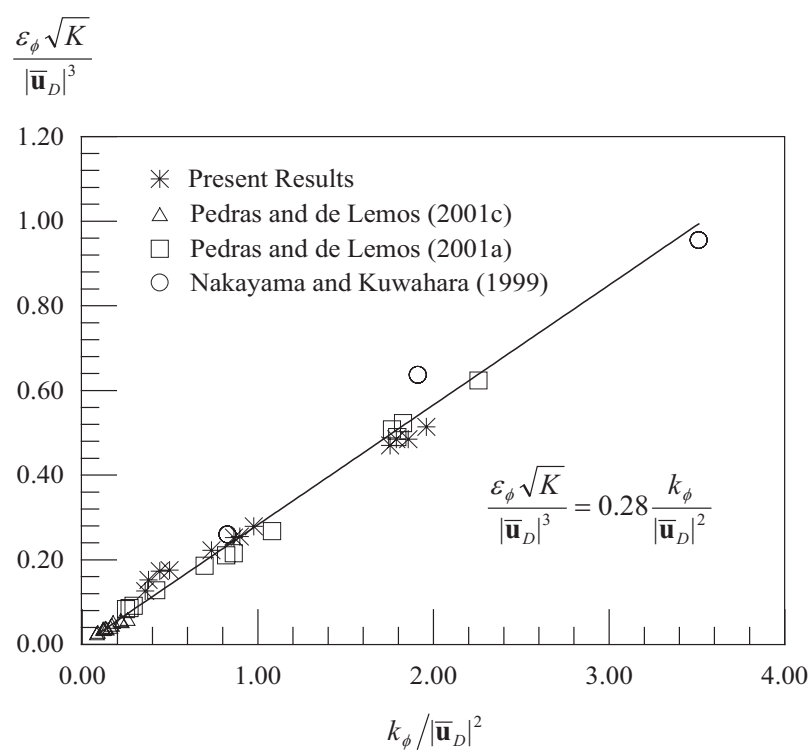

Fig. 4 - Determination of value for $c_{k}$ using data for different porous media, porosity and Reynolds number.

\section{MACROSCOPIC MODEL RESULTS}

With the numerical evaluation of $c_{k}$, calculations using the macroscopic turbulence model above can be performed. A test case consisting in simulating the flow through a porous bed of length $10 \mathrm{H}$, starting with a pre-selected initial conditions greater than the final asymptotic values, is now carried out. Similar test results were reported by Pedras and de Lemos (2001a,c) and Nakayama and Kuwahara (1999) being the values at entrance $\langle k\rangle^{i}=10 k_{\phi}$ and $\langle\varepsilon\rangle^{i}=30 \varepsilon_{\phi}$. Figures 5 and 6 show results for $\langle k\rangle^{i}$ and $\langle\varepsilon\rangle^{i}$ along the flow. Calculations are compared with similar results of Nakayama and Kuwahara (1999) for an array of square rods and with computations of Pedras and de Lemos (2001a,c) for a bed of cylindrical and longitudinal elliptic rods, respectively. Is interesting to note that, in spite of differences on the shape of the rods, the axial decay is nearly the same in all cases indicating the coherence of the results herein with previous data published in the literature. 


\section{CIÊNCIA/SCIENCE}

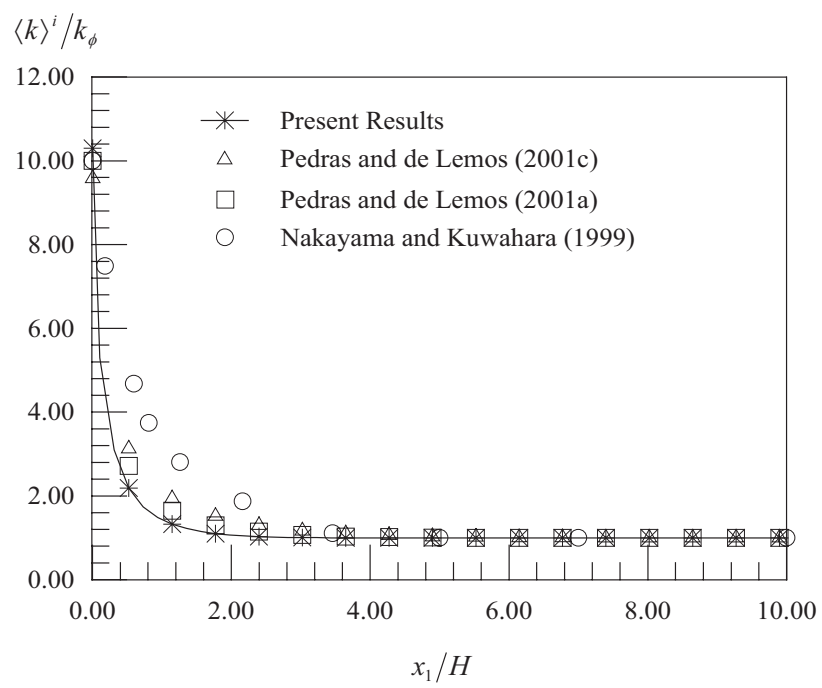

Fig. 5 - Development of non-dimensional turbulence kinetic energy.

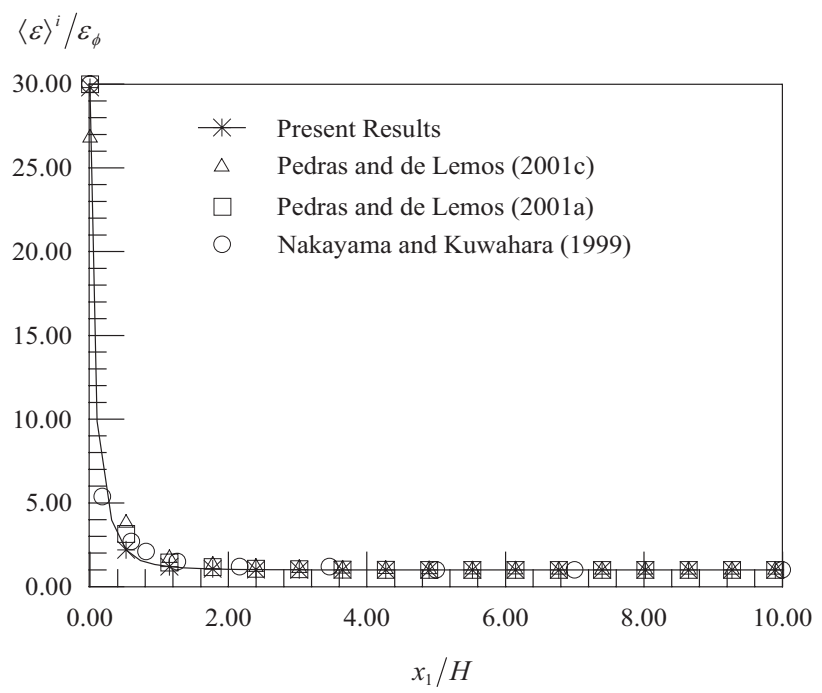

Fig. 6 - Development of non-dimensional dissipation rate.

\section{CONCLUSIONS}

A macroscopic turbulence model was adjusted for an infinite porous medium formed by spatially periodic array of transversal elliptic rods. This adjustment was carried out by the solution of the microscopic flow field within the volume occupied by the fluid. After that, integrated flow properties were computed and the proposed model constant was determined. Then, the macroscopic model was tested comparing the numerical results of the flow in the entrance region of homogeneous isotropic porous medium with available similar results in the recent literature. Good agreement with publishing data was observed.

\section{ACKNOWLEDGEMENTS}

The authors are thankful to FAPESP and CNPq, for their financial support during the course of this research.

\section{NOMENCLATURE}

$c_{k}, c_{1 \varepsilon}, c_{2 \varepsilon}, c_{\mu}$ dimensionless constants

$C_{1}, C_{2}, C_{\mu} \quad$ dimensionless constants

$f_{2}, f_{\mu}$ damping functions

$H \quad$ characteristic length (Fig. 2)

$k \quad$ turbulence kinetic energy

$\langle k\rangle^{i} \quad$ intrinsic (fluid) average of $k$

$k_{\phi} \quad$ fully developed value of $\langle k\rangle^{i}$

u microscopic velocity vector

$\overline{\mathbf{u}}_{D} \quad$ Darcy velocity vector

$u, v$ components of $\mathbf{u}$

Greek symbols

$\varepsilon \quad$ dissipation rate of $k$

$\langle\varepsilon\rangle^{i} \quad$ intrinsic (fluid) average of $\varepsilon$

$\varepsilon_{\phi} \quad$ fully developed value of $\langle\varepsilon\rangle^{i}$

$\mu \quad$ dynamic viscosity

$\mu_{t_{\phi}} \quad$ macroscopic coefficient of exchange for porous media

$\mu_{t} \quad$ turbulent viscosity

$\sigma_{k}, \sigma_{\varepsilon}$ effective Prandtl number

$\rho \quad$ fluid density

$\phi \quad$ porosity 


\section{REFERENCES}

Abe, K., Nagano, Y., and Kondoh, T., 1992, “An Improved $\mathrm{k}-\varepsilon$ Model for Prediction of Turbulent Flows with Separation and Reattachment". Trans. JSME, Ser. B, vol. 58, pp. 3003-3010.

Antohe, B. V. and Lage, J. L., 1997, "A General Two-Equation Macroscopic Turbulence Model for Incompressible Flow in Porous Media". Int. J. Heat Mass Transfer, vol. 40, pp. 3013-3024.

Bear, J., 1972, "Dynamics of Fluids in Porous Media”. American Elsevier Pub. Co., New York.

Brinkman, H. C., 1947, "A Calculation of the Viscous Force Exerted by a Flowing Fluid on a Dense Swarm of Particles". Appl. Sci. Research, A 1, pp. 27-34.

Darcy, H., 1856, "Les Fontaines Publiques de La Vile de Dijon". Victor Dalmond, Paris.

de Lemos, M. J. S. and Pedras, M. H. J., 2000, "Modeling turbulence Phenomena in Incompressible Flow Through Saturated Porous Media”. 34th ASME-NHTC, paper NHTC2000-12120, ISBN 0-7918-1997-3.

de Lemos, M. J. S. and Pedras, M. H. J., 2001, "Recent Mathematical Models for Turbulent Flow in Saturated Rigid Porous Media". ASME Journal of Fluids Engineering, vol. 123 (4), pp. 935-940.

Forchheimer, P., 1901, "Wasserbewegung Durch Boden”. Z. Ver. Deutsch. Ing., vol. 45, pp. 1782-1788.

Hsu, C. T. and Cheng, P., 1990, "Thermal Dispersion in a Porous Medium”. Int. J. Heat Mass Transfer, vol. 33, pp. 1587-1597.

Kececiooglu, I. and Jiang, Y., 1994, "Flow Through Porous Media of Packed Spheres Saturated with Water". J. Fluids Engineering, vol. 116, pp. 164-170.

Kuwahara, F., Kameyama, Y., Yamashita, S., and Nakayama, A., 1998, "Numerical Modeling of Turbulent Flow in Porous Media Using a Spatially Periodic Array". J. Porous Media, vol. 1, pp. 47-55.

Launder, B. E. and Spalding, D. B., 1974, "The Numerical Computation of Turbulent Flows". Comp. Meth. Appl. Mech. Eng., vol. 3, pp. 269-289.

Macdonald, I. F., El-Sayed, M. S., Mow, K., and Dullien, F. A. L., 1979, "Flow Through Porous Media: The Ergun Equation Revisited". Indust. Chem. Fundam., vol. 18, pp. 199-208.

Nakayama, A. and Kuwahara, F., 1999, “A Macroscopic Turbulence Model for Flow in a Porous Medium". ASME Journal of Fluids Engineering, vol. 121, pp. 427-433.

Patankar, S. V., 1980, "Numerical Heat Transfer and Fluid Flow". Hemisphere, New York.

Pedras, M. H. J. and de Lemos, M. J. S., 1998, "Analysis of the Flow in Porous Media via Volumetric Averaging of the Navier-Stokes Equations". Proc. of ENCIT98 $7^{\text {th }}$ Braz. Therm. Scienc. Meetg. (Rio de Janeiro, Brazil). In Portuguese.

Pedras, M. H. J. and de Lemos, M. J. S., 1999, “On Volume and Time Averaging of Transport Equations for Turbulent Flow in Porous Media". ASME-FED-vol. 248, Paper FEDSM99-7273, ISBN 0-7918-1961-2.

Pedras, M. H. J. and de Lemos, M. J. S., 2000, “On the Definition of Turbulent Kinetic Energy for Flow in
Porous Media". Intern. Comm. Heat and Mass Transfer, vol. 27 (2) pp. 211-220.

Pedras, M. H. J. and de Lemos, M. J. S., 2001a, "Macroscopic Turbulence Modeling for Incompressible Flow Through Undeformable Porous Media". Int. J. Heat Mass Transfer, vol. 44 (6), pp. 1081-1093.

Pedras, M. H. J. and de Lemos, M. J. S., 2001b, "Simulation of Turbulent Flow in Porous Media Using a Spatially Periodic Array and a Low Re Two-Equation Closure". Numer. Heat Transfer Part A-Appl, vol. 39 (1), pp. 35-59.

Pedras, M. H. J. and de Lemos, M. J. S., 2001c, "Adjustment of a Macroscopic Turbulence Model for a Porous Medium Formed by an Infinite Array of Elliptic Rods". Proc. of $2^{\text {nd }}$ Int. Conf. Computational Heat and Mass Trnas. (Rio de Janeiro, Brazil).

Pedras, M. H. J. and de Lemos, M. J. S., 2001d, "On Mathematical Description and Simulation of Turbulent Flow in a Porous Medium Formed by an Array of Elliptic Rods". ASME Journal of Fluids Engineering, vol. 123 (4), pp. 941-947.

Rocamora Jr, F. D. and de Lemos. M. J. S., 1998, "Numerical Solution of Turbulent Flow in Porous Media Using a Spatially Periodic Array and The k- $\varepsilon$ Model". Proc. of ENCIT98- $7^{\text {th }}$ Braz. Therm. Scienc. Meetg. (Rio de Janeiro, Brazil).

Takatsu, Y. and Masuoka, T., 1998, "Turbulent Phenomena in Flow Through Porous Media". J. Porous Media, vol. 3, pp. 243-251.

Vafai, K. and Tien, C. L., 1981, "Boundary and Inertia Effects on Flow and Heat Transfer in Porous Media”. Int. J. Heat Mass Transfer, vol. 24, pp. 195-203.

Ward, J. C., 1964, "Turbulent Flow in Porous Media”. J. Hydraul. Div. ASCE, vol. 90 (HY5), pp. 1-12.

Whitaker, S., 1969, "Advances in Theory of Fluid Motion in Porous Media". Indust. Engng. Chem., vol. 61, pp. 14-28. 